\title{
Sham Intervention
}

National Cancer Institute

\section{Source}

National Cancer Institute. Sham Intervention. NCI Thesaurus. Code C116527.

A procedure that functions as the control arm of a clinical trial, mimicking the procedure under investigation in every way, including the preprocedure routine, anaesthesia and incisions (if necessary), and post-procedure follow-up, without actually providing or performing the procedure being studied. 